\title{
Radioablación por laparoscopia de masas renales pequeñas en pacientes octogenarios: técnica paso a paso
}

\section{Laparoscopic Radioablation of Small Renal Masses in Octogenarian Patients: Step by Step Technique}

\author{
Natalia Bautista Delgado ${ }^{1}$ Jorge A. Forero ${ }^{2}$ Rodolfo Varela ${ }^{2}$ Marino Cabrera F. ${ }^{2}$ \\ 1 Postgrado de Urología, Fundación Universitaria Sanitas, Bogotá, Colombia \\ ${ }^{2}$ Departamento de Urología Oncológica, Instituto Nacional de \\ Cancerología, Ciudad de México, Mexico \\ Address for correspondence Natalia Bautista Delgado, MD, Clínica \\ Universitaria Colombia, Bogotá, Colombia \\ (e-mail: nbautistad1028@gmail.com).
}

Urol Colomb 2018;27:290-293.

\section{Resumen \\ Palabras Clave \\ - tumores renales pequeños \\ - ablación por radiofrecuencia \\ - nefrectomía parcial \\ - cirugía conservadora de nefronas \\ - supervivencia libre de enfermedad}

Introducción La cirugía preservadora de nefronas sigue siendo el gold standard de manejo en pacientes con tumores renales pequeños. La ablación por radiofrecuencia es una alternativa mínimamente invasiva con evidencia sustancial en efectividad en la erradicación tumoral completa.

Objetivo Reportar el caso y la técnica paso a paso de la radioablación laparoscópica de un tumor renal en un centro de oncología de III nivel de Bogotá - Colombia.

Materiales y Métodos Revisión de la historia clínica de un paciente con hallazgo incidental de una masa renal pequeña sometido a radioablación laparoscópica en un centro de oncología de III nivel de Bogotá.

Conclusión La ablación por radiofrecuencia es una alternativa válida para el tratamiento de tumores renales pequeños, especialmente en adultos mayores y con alto riesgo quirúrgico, ofreciendo tasas de supervivencia libre de enfermedad comparables con la nefrectomía parcial.

Introduction Nephron-sparing surgery remains the gold standard of management in patients with small renal tumors. Radiofrequency ablation is a minimally invasive alternative with substantial evidence of effectiveness in complete tumor eradication. Objective To describe a case and laparoscopic radioablation step by step technique of a renal tumor in an oncology center of III level in Bogotá - Colombia.

Materials and Methods Review of the clinical history of a patient with an incidental finding of small renal mass undergoing laparoscopic radioablation at a level III oncology center in Bogotá.

Conclusion Radiofrequency ablation is a valid alternative for the treatment of small renal tumors, especially in older adults with high surgical risk, offering disease free survival rates comparable to partial nephrectomy. received

April 7, 2017

accepted

November 17, 2017

published online

May 29, 2018
DOI https://doi.org/

$10.1055 / \mathrm{s}-0038-1656518$.

ISSN 0120-789X.

eISSN 2027-0119.
Copyright (c) 2018, Sociedad Colombiana License terms de Urología. Publicado por Thieme Revinter Publicações Ltda., Rio de Janeiro, Brazil. Todos los derechos reservados.

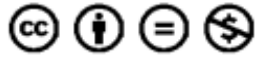




\section{Introducción}

El hallazgo incidental de tumores renales T1a se ha incrementado en la última década. ${ }^{1}$ La cirugía preservadora de nefronas sigue siendo el gold standard de manejo en pacientes con tumores renales pequeños, con resultados oncológicamente equiparables a la nefrectomía radical y reduciendo el impacto negativo en la función renal. $^{2,3}$ La ablación por radiofrecuencia (RFA), es una alterativa mínimamente invasiva cuya técnica causa la destrucción del tumor mediante el calentamiento. ${ }^{4,5}$ Puede ser realizada mediante cirugía abierta o laparoscópica y por vía percutánea guiada por tomografía axial o ecografía. La evidencia sustancial indica que la RFA de tumores renales pequeños es efectiva en la erradicación tumoral completa. ${ }^{6-8}$

\section{Objetivo}

Reportar el caso clínico y la técnica quirúrgica paso a paso de la radioablación laparoscópica de un tumor renal en un centro de oncología de III nivel de Bogotá.

\section{Materiales y Métodos}

Reportamos el caso de un paciente masculino de 81 años con hallazgo incidental de tumor renal izquierdo.

\section{Antecedentes}

El paciente tiene como antecedentes personales hipertensión arterial, enfermedad pulmonar obstructiva crónica con suplencia de oxígeno las 24 horas y extabaquismo pesado. Paciente asintomático urinario, sin episodios previos de hematuria o algún antecedente urológico de importancia.

\section{Hallazgos Clínicos}

Al examen físico no se documentan masas con la palpación abdominal, sin dolor, sin irritación peritoneal. El resto del examen físico, se encuentra dentro de los límites normales.

\section{Evaluación Diagnóstica}

El paciente se encuentra adscrito al programa de enfermedades crónicas de su unidad primaria de atención en salud. Durante los chequeos rutinarios al realizar una ecografía renal y de vías urinarias, se documenta una lesión con aparente densidad de tejidos blandos de $33 \mathrm{~mm} \times 28 \mathrm{~mm}$ dependiente del polo renal superior izquierdo. Por esa razón, se decide complementar dicho hallazgo con una imagen abdominal total contrastada. La tomografía revela una masa exofítica de $4 \mathrm{~cm}$ de diámetro dependiente de polo renal superior izquierdo (-Fig. 1). Se clasifica entonces como un tumor renal izquierdo cT1aNxM0. Debido a sus comorbilidades de base se considera paciente con alto riesgo quirúrgico y por lo tanto se define radioablación percutánea del tumor renal. El paciente es valorado por el servicio de radiología intervencionista quienes al evaluar las imágenes documentan la presencia de colon retro renal que condiciona dificultad para el acceso percutáneo. El paciente es remitido nuevamente a Urología para manejo laparoscópico de la lesión.

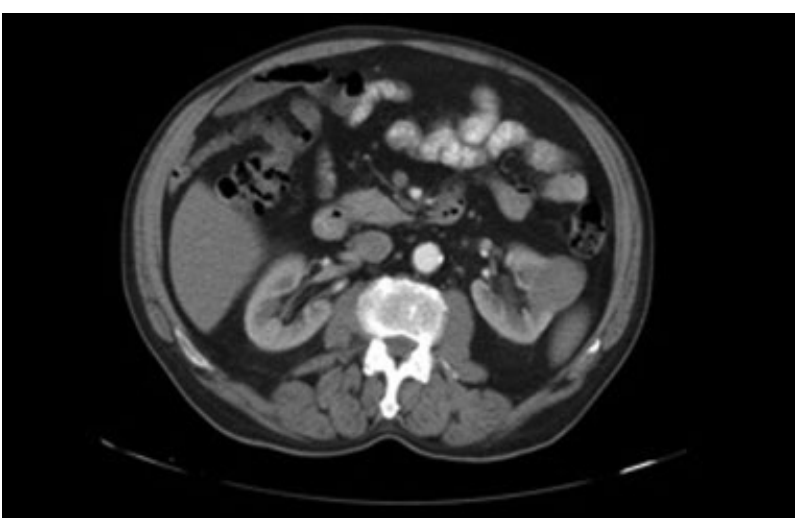

Fig. 1 TAC abdominal en donde se documenta masa renal de $4 \mathrm{~cm}$.

\section{Técnica Quirúrgica}

El paciente es sometido al procedimiento quirúrgico bajo anestesia general, monitorización mediante línea arterial y se instaura acceso venoso yugular externo. Se realiza paso de sonda uretral de forma rutinaria para la cuantificación de la diuresis. Posteriormente, se ubica en posición de decúbito lateral derecho con protección de áreas de presión como el plexo braquial sin necesidad de posición en flex.

Se procede a realizar inserción de los puertos de laparoscopia, iniciando con el puerto de $10 \mathrm{~mm}$ pararrectal izquierdo a nivel supraumbilical. Se inicia neumoperitoneo y posteriormente, bajo visión directa, se realiza paso de dos puertos de trabajo así: uno inferior de $10 \mathrm{~mm}$ a nivel de la fosa ilíaca izquierda y uno superior de $5 \mathrm{~mm}$ subcostal izquierdo. Con abordaje transperitoneal se procede a realizar medialización del colon izquierdo hasta lograr exposición de la masa descrita, apertura de la grasa perirrenal y exéresis de la grasa peritumoral. Una vez aislada la masa renal, se realiza paso de transductor ecográfico laparoscópico a través del puerto de $10 \mathrm{~mm}$. Se procede con la toma de ecografía intraoperatoria documentando la profundidad de la masa, su relación con el sistema colector y evaluación de masas adicionales (-Figs. 2A y B). Paso de aguja trucut y toma de 3 biopsias de la lesión bajo visión directa (-Fig. 3). Paso de agujas de radiofrecuencia (-Fig. 4A), toma de nuevas medidas y a través del servicio técnico de radiofrecuencia se procede a la ubicación del extremo distal de la aguja de $\operatorname{Rita}^{\mathrm{R}}$ a $6 \mathrm{~mm}$ del centro del tumor (-Figs. 4B y C). Se inicia la radioablación durante 13 minutos con potencia de $150 \mathrm{~W}$, enfriamiento a $-60^{\circ} \mathrm{C}$ y nueva ablación con los parámetros descritos alcanzando 26 minutos en total y $105^{\circ} \mathrm{C}$ de temperatura ( - Figs. 5A y B). Se realiza una pausa de 10 minutos con tensión arterial media del paciente mayor a $70 \mathrm{~mm} \mathrm{Hg}$ verificando presencia de sangrado residual. El tiempo operatorio fue de 60 minutos, el sangrado estimado fue de $50 \mathrm{~mL}$. No se presentaron complicaciones perio o postoperatorias. El paciente no requirió paso de dren ni monitorización en cuidados intensivos. El paciente evolucionó satisfactoriamente y es dado de alta al segundo día del postoperatorio.

El primer control ambulatorio es llevado a cabo 8 días posteriores a la intervención evidenciando una adecuada evolución clínica. El estudio histopatológico informó 

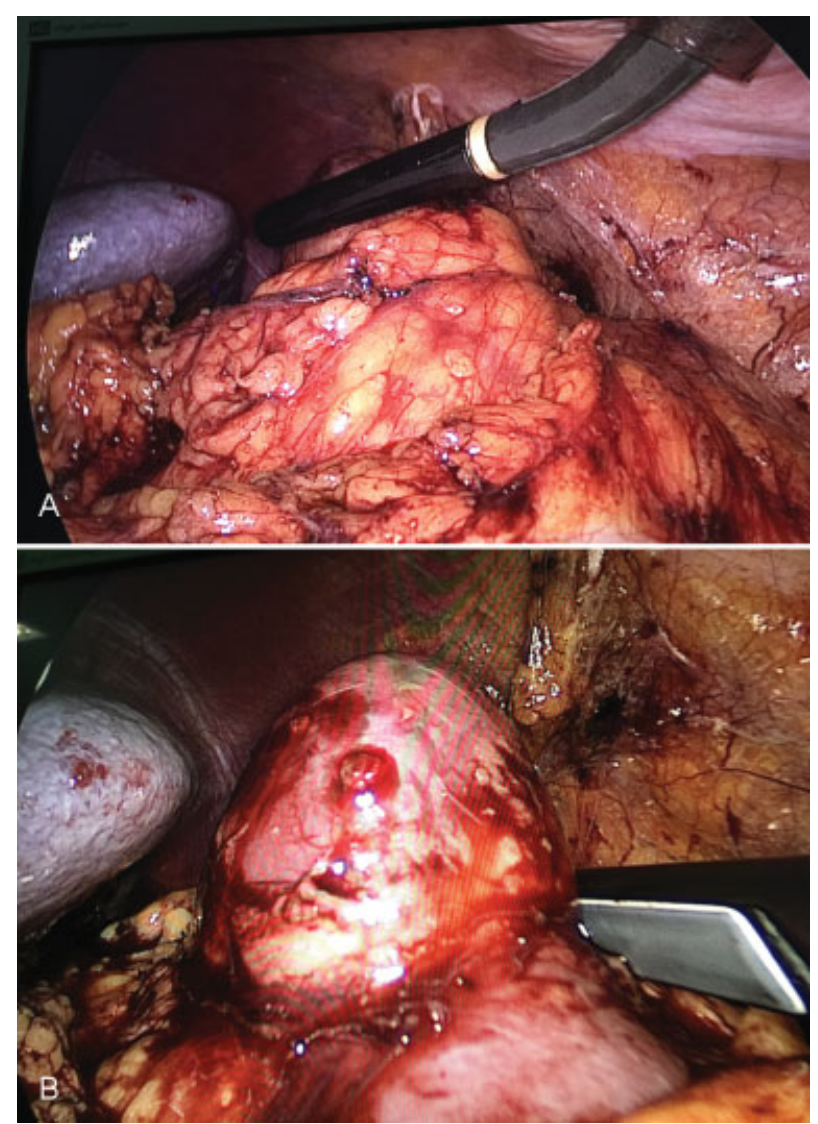

Fig. 2 (A) Ecografía intraoperatoria. (B) Evaluación de la profundidad de la lesión.

carcinoma de células renales cromófobo. Se realizó control tomográfico semestral en donde no se documenta recaída tumoral o aumento en la lesión (-Fig. 6).

\section{Discusión}

La cirugía preservadora de nefronas es el gold standard de manejo en pacientes con tumores renales pequeños; ${ }^{1}$ sin embargo, algunos pacientes no son candidatos ideales para una resección quirúrgica invasiva debido al gran número de comorbilidades de base. Las técnicas ablativas mínimamente invasivas como la radiofrecuencia, pueden ser realizadas

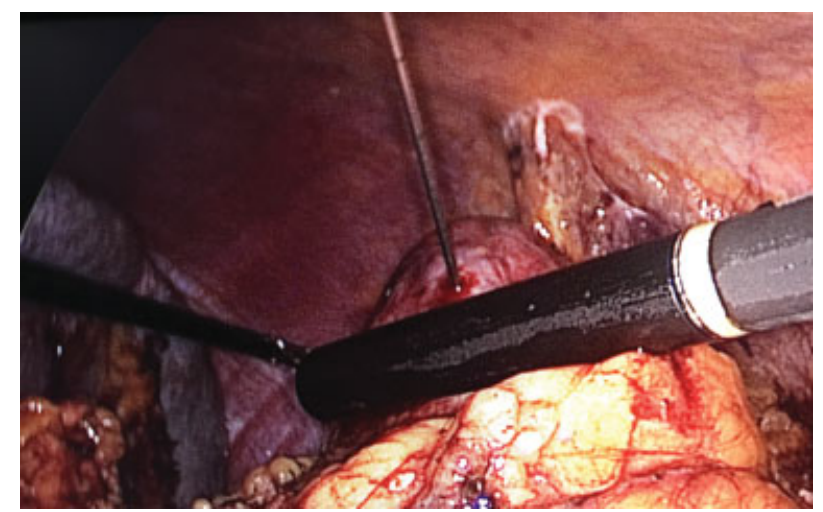

Fig. 3 Toma de biopsias de la lesión.
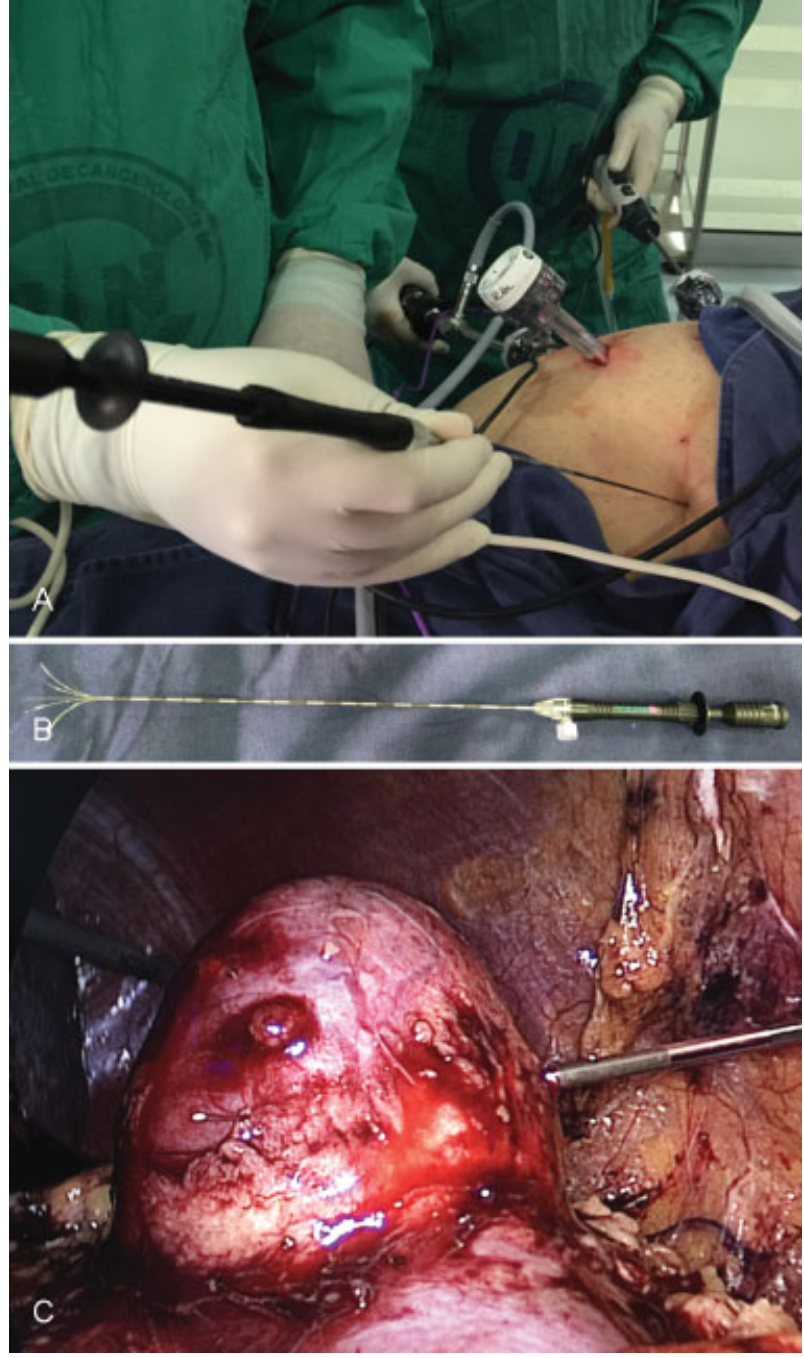

Fig. 4 (A) Paso percutáneo de Aguja Rita. (B) Aguja Rita. (C) Ubicación de la aguja en el centro del tumor.

mediante cirugía abierta o laparoscópica y por vía percutánea guiada por tomografía axial o ecografía $a^{3,5}$ y han sido descritas en la literatura actual como un tratamiento seguro y efectivo para los tumores renales $\mathrm{cT} 1 \mathrm{a} / \mathrm{b}$ con resultados oncológicos comparables con la nefrectomía parcial. ${ }^{9}$ En el meta-análisis realizado por Xu y col. en 2015, comparando la radiofrecuencia vs nefrectomía parcial, demostró que la radiofrecuencia es una técnica conservadora de nefronas menos invasiva, indicada en pacientes con alto riesgo quirúrgico dado por una falla renal instaurada, pacientes monorrenos o con comorbilidades como enfermedad coronaria, cardiomiopatía o enfermedad pulmonar obstructiva. Dado lo anterior, concluyen que la ablación por radiofrecuencia es ideal para los pacientes con edad avanzada, con una incidencia de complicaciones de 6,3\% para la radiofrecuencia vs $29,4 \%$ para la nefrectomía parcial, sin alcanzar una significancia estadística (OR: 0,74 CI: 0,35-1,55), y con una menor estancia hospitalaria con una diferencia promedio de 2.02 días. $^{10}$

El planeamiento previo al procedimiento con estudios imagenológicos (tomografía o ultrasonido), es esencial para detectar aquellas que requieren especial cuidado para 

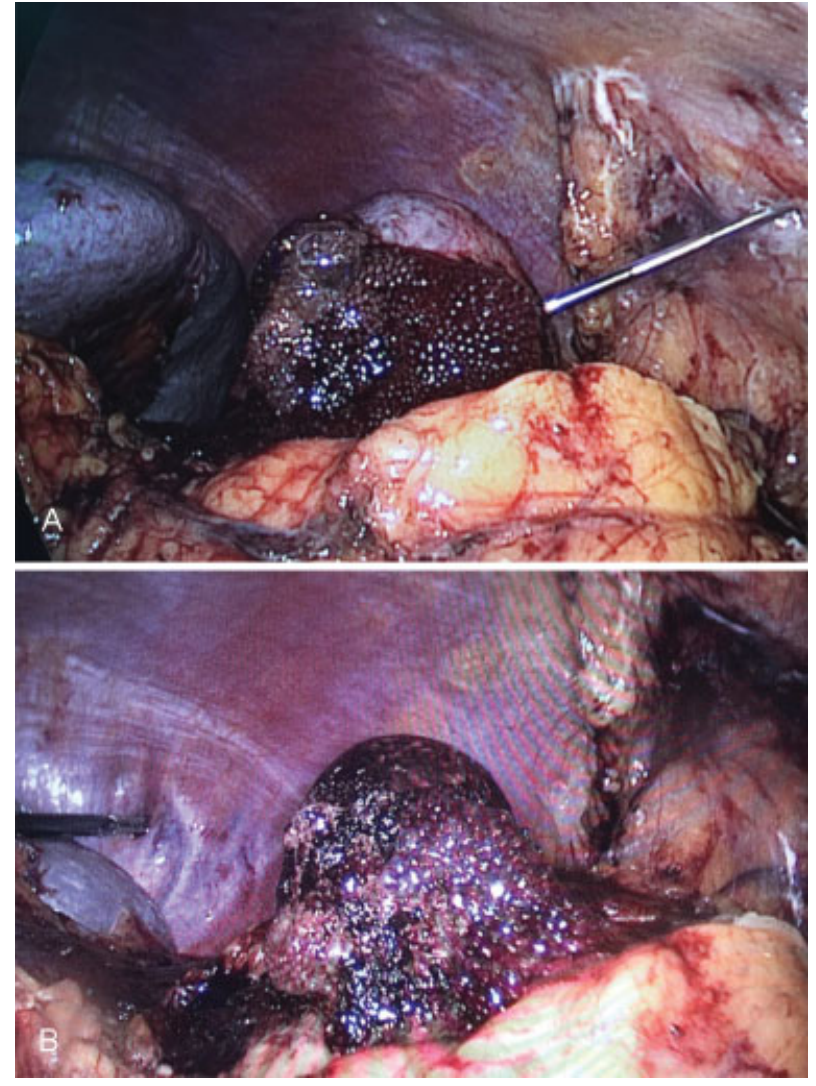

Fig. 5 (A) Radioablación y enfriamiento. (B) Resultado final.

minimizar el riesgo de lesión térmica (masas cercanas a la vía urinaria o estructuras digestivas) ${ }^{11}$ La realización de biopsia en el mismo acto quirúrgico de la radioablación ofrece múltiples ventajas incluyendo la disminución del riesgo de sangrado masivo post biopsia debido a que la ablación del tumor reduce por completo el flujo sanguíneo. ${ }^{3}$

\section{Conclusión}

La ablación por radiofrecuencia es una alternativa válida para el tratamiento de tumores renales pequeños, especialmente en adultos mayores y con alto riesgo quirúrgico, ofreciendo

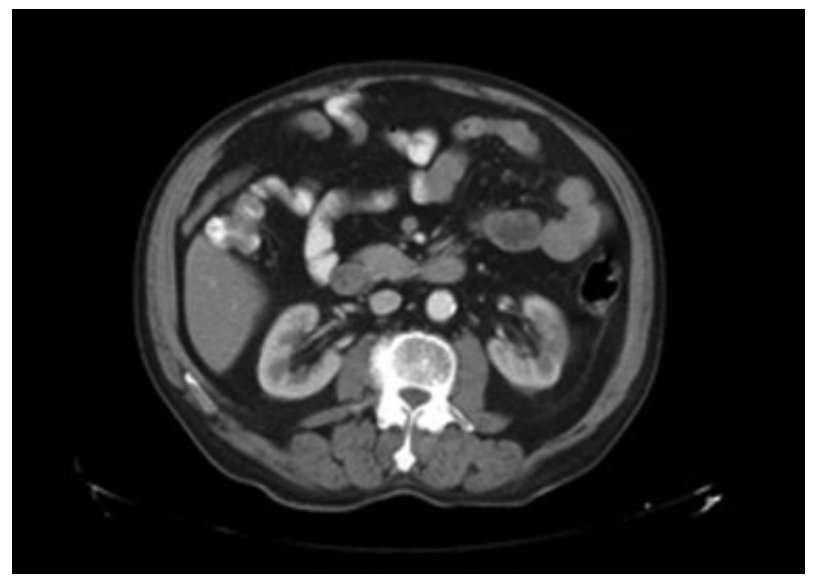

Fig. 6 TAC abdominal de seguimiento a los 6 meses. tasas de supervivencia libre de enfermedad comparables con la nefrectomía parcial.

\section{Responsabilidades Éticas}

Protección de personas y animales. Los autores declaran que para esta investigación no se han realizado experimentos en seres humanos ni en animales.

Confidencialidad de los datos. Los autores declaran que han seguido los protocolos de su centro de trabajo sobre la publicación de datos de pacientes.

Derecho a la privacidad y consentimiento informado. Los autores declaran que en este artículo no aparecen datos de pacientes.

\section{Financiación}

La financiación del presente trabajo fue asumida en su totalidad por los autores.

Conflicto de Interés

Los autores declaran que no tienen ningún conflicto de interés.

\section{Bibliografía}

1 Wha TM, Irving HC, Gregory W, Cartledge J, Joyce AD, Selby PJ. Radiofrequency ablation (RFA) of renal cell carcinoma (RCC): experience in 200 tumours. BJU Int 2014;113(03):416-428

2 Kelly EF, Leveillee RJ. Image guided radiofrequency ablation for small renal masses. Int J Surg 2016;36(Pt C):525-532

3 Iguchi T, Hiraki T, Tomita K, et al. Simultaneous biopsy and radiofrequency ablation of T1a renal cell carcinoma. Diagn Interv Imaging 2016;97(11):1159-1164

4 Morgan MS, Ozayar A, Lucas E, Friedlander JI, Shakir NA, Cadeddu JA. Comparative effects of irreversible electroporation, radiofrequency ablation, and partian nephrectomy on renal function preservation in a porcine solitary kidney model. Urology 2016;94:281-287

5 Alguersuari A, Mateos A, Falcó J, Criado E, Fortuño JR, Guitart J. Ablación percutánea mediante radiofrecuencia de tumores renales en pacientes de alto riesgo: 10 años de experiencia. Radiologia 2016;58(05):373-379

6 Forauer AR, Dewey BJ, Seigne JD. Cancer-free survival and local tumor control after impendence-based radiofrequency ablation of biopsy-proven renal cell carcinomas with a minimum of 1-year follow-up. Urol Oncol 2014;32(06):869-876

7 Hoffmann RT, Jakobs TF, Kubisch CH, et al. Renal cell carcinoma in patients with a solitary kidney after nephrectomy treated with radiofrequency ablation: mid term results. Eur J Radiol 2010;73 (03):652-656

8 Mylona S, Kokkinaki A, Pomoni M, Galani P, Ntai S, Thanos L. Percutaneous radiofrequency ablation of renal cell carcinomas in patients with solitary kidney: 6 years experience. Eur J Radiol 2009;69(02):351-356

9 Liu N, Huang D, Cheng X, et al. Percutaneous radiofrequency ablation for renal cell carcinoma vs. partial nephrectomy: Comparison of long-term oncologic outcomes in both clear cell and non-clear cell of the most common subtype. Urol Oncol 2017; 35(08):530.e1-530.e6

10 Pan XW, Cui XM, Huang H, et al. Radiofrequency ablation versus partial nephrectomy for treatment of renal masses: A systematic review and meta-analysis. Kaohsiung J Med Sci 2015;31(12):649-658

11 Trilla E, Konstantinidis C, Serres X, et al. Radiofrecuencia percutánea guiada por ecografía en el tratamiento de masas renales pequeñas. Actas Urol Esp 2017;41(08):497-503 